\title{
In-Flow and Out-Flow Problem for the Stokes System
}

\author{
Bernard Nowakowski(i) and Gerhard Ströhmer \\ Communicated by Y. Giga
}

\begin{abstract}
We investigate the existence and regularity of solutions to the stationary Stokes system and non-stationary NavierStokes equations in three dimensional bounded domains with in- and out-lets. We assume that on the in- and out-flow parts of the boundary the pressure is prescribed and the tangential component of the velocity field is zero, whereas on the lateral part of the boundary the fluid is at rest.
\end{abstract}

Mathematics Subject Classification. Primary 35B65, 35Q30, 76D03; Secondary 76D05.

Keywords. In- and out-flow, Stokes system, Dynamic boundary conditions.

\section{Introduction}

Let us consider the Stokes system

$$
\begin{array}{ll}
-\nu \Delta \mathbf{u}+\nabla \Pi=\mathbf{f} & \text { in } \Omega, \\
\operatorname{div} \mathbf{u}=g & \text { in } \Omega,
\end{array}
$$

where $\Omega \subset \mathbb{R}^{3}$ is a bounded solid with piecewise $\mathcal{C}^{2}$-boundary $\Gamma:=\partial \Omega$. Functions $\mathbf{u}$ (the velocity) and $\Pi$ (the pressure) are unknown. Given are $\mathbf{f}$ (the external force), $g$ and a positive viscosity coefficient $\nu$. We are interested in the existence and regularity of solutions $(\mathbf{u}, \Pi)$ to $(1)$ when $\Omega$ has cylindrical in- and outlets. More specifically, we assume that $\Gamma$ is the union of $\Gamma_{\text {in,out }}$ and $\Gamma_{\text {lat }}$, where $\Gamma_{\text {in,out }}=\bigcup_{1 \leq k \leq N} \Gamma_{\text {in,out }}^{k}$. We also assume that for all $1 \leq k \leq N$ the surfaces $\Gamma_{i n, o u t}^{k}$ are flat and

$$
\Gamma_{\text {in,out }}^{k} \text { is orthogonal to } \Gamma_{l a t}
$$

(see Fig. 1). The latter assumption ensures that we do not need to work in the framework of weighted Sobolev spaces and can rely on the reflection principle.

We need to supplement (1) with proper boundary conditions. On $\Gamma_{\text {lat }}$ we assume that the fluid is at rest. The choice of the boundary conditions on $\Gamma_{i n, o u t}^{k}$ depends on the problem we would like to model. For some examples of such problems and further motivations originating from real-life situations we refer the reader to the Introduction in e.g. [1,2] and [3, Sect. 3]. The most frequently used boundary conditions are:

- prescribed pressure drops (see e.g. $[3,4])$

$$
\frac{1}{\left|\Gamma_{\text {in }, \text { out }}^{k}\right|} \int_{\Gamma_{i n, \text { out }}^{k}} \Pi \mathrm{d} S=\Pi_{k}(t)
$$

where $\Pi_{k}(t)$ are given functions and $\left|\Gamma_{i n, o u t}^{k}\right|$ denotes the surface area of $\Gamma_{\text {in,out }}^{k}$,

- prescribed net fluxes (see e.g. $[3,5,6]$ )

$$
\int_{\Gamma_{i n, o u t}^{k}} \mathbf{u} \cdot \mathbf{n} \mathrm{d} S=F_{k}(t),
$$




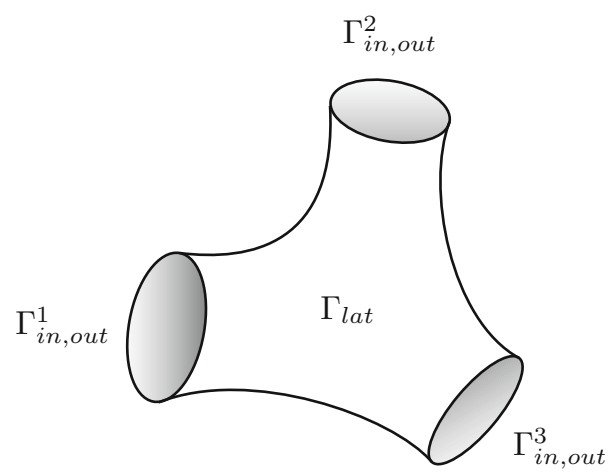

FIG. 1. An example of $\Omega$ when $N=3$

where $F_{k}(t)$ are given functions,

- prescribed pressure and tangential components of the velocity (see e.g. [1,2])

$$
\begin{aligned}
\left.\Pi\right|_{\Gamma_{i n, \text { out }}^{k}} & =\Pi_{k}, \\
\mathbf{v} \times \mathbf{n} & =\mathbf{a}_{k} \times \mathbf{n},
\end{aligned}
$$

where $\Pi_{k}$ and $\mathbf{a}_{k}$ are given functions.

Other boundary conditions that appear in the literature include the so-called artificial boundary conditions (ABCs, see e.g. [7,8]), prescribed normal components of the velocity field on $\Gamma_{\text {in out }}^{k}$ (see e.g. $[9,10]$ ), modified do-nothing boundary conditions (see e.g. [11,12]) and other (see e.g. [13-15]). This list, however, is far from being complete but forms a good starting point for further research.

In our work we consider (2) with $\mathbf{a}_{k}=\mathbf{0}$. It can be rewritten as

$$
\begin{array}{ll}
\mathbf{u}_{\text {tan }}=\mathbf{0} & \text { on } \Gamma, \\
\Pi=\Pi_{\text {in,out }}^{k} & \text { on } \Gamma_{\text {in }, \text { out }}^{k}, \\
\left.\mathbf{u} \cdot \mathbf{n}\right|_{\Gamma_{\text {lat }}} ^{k}=0 & \text { on } \Gamma_{\text {lat }},
\end{array}
$$

where $\mathbf{u}_{\text {tan }}$ denotes the tangential component of $\mathbf{u}$. We will justify our choice after Theorem 2 . Our main result reads:

Theorem 1. Suppose that $\mathbf{f} \in L_{2}(\Omega), g \in H^{1}(\Omega), \Pi_{i n, \text { out }}^{k} \in H^{\frac{1}{2}}\left(\Gamma_{\text {in out }}^{k}\right)$ and $\left.g\right|_{\Gamma_{\text {in out }}^{k}}=0$. Then, there exists a unique solution $(\mathbf{u}, \Pi) \in H^{2}(\Omega) \times H^{1}(\Omega)$ to $(1)+(3)$ such that

$$
\begin{aligned}
& \|\mathbf{u}\|_{H^{2}(\Omega)}+\|\Pi\|_{H^{1}(\Omega)} \\
& \quad \leq c\left(\frac{1}{\nu}, \Omega\right)\left(\|\mathbf{f}\|_{L_{2}(\Omega)}+\|g\|_{H^{1}(\Omega)}+\sum_{k}\left\|\Pi_{\text {in out }}^{k}\right\|_{H^{\frac{1}{2}\left(\Gamma_{\text {in out }}^{k}\right)}}\right) .
\end{aligned}
$$

Problem $(1)+(3)$ with $g=0$ was already considered in $[1,2,16-18]$. In these papers the authors assume that $\Gamma=\Gamma_{1} \cup \Gamma_{2} \cup \Gamma_{3}$. Comparing with our notation we see that $\Gamma_{1}=\Gamma_{\text {lat }}$ and $\Gamma_{2}=\bigcup_{k} \Gamma_{\text {in,out }}^{k}$. On $\Gamma_{1}$ and $\Gamma_{2}$ slightly more general boundary conditions are assumed, namely $\left.\mathbf{u}\right|_{\Gamma_{1}}=\mathbf{u}_{0}$ and $\left.\mathbf{u}_{t a n}\right|_{\Gamma_{2}}=\mathbf{a}$, where both $\mathbf{u}_{0}$ and $\mathbf{a}$ are given. On $\Gamma_{3}$ in these papers we have

$$
\operatorname{rot} \mathbf{u} \times \mathbf{n}=\mathbf{h} \times \mathbf{n}, \quad \mathbf{u} \cdot \mathbf{n}=\mathbf{b} \cdot \mathbf{n},
$$

where $\mathbf{h}$ and $\mathbf{b}$ are prescribed. Assuming that $\mathbf{u}_{0} \in H^{\frac{1}{2}}\left(\Gamma_{1}\right), \mathbf{a} \in H^{\frac{1}{2}}\left(\Gamma_{2}\right), \mathbf{b} \in H^{\frac{1}{2}}\left(\Gamma_{3}\right)$ and $\mathbf{h} \in H^{-\frac{1}{2}}\left(\Gamma_{3}\right)$ the existence of $\mathbf{u}$ in $H^{1}(\Omega)$ is shown. Under slightly stronger assumptions on the boundary data the $H^{2}$-regularity of the solutions was obtained in [19]. Theorem 1 is similar to [19, Theorem 1.2] but the proofs are very different. Instead of constructing variational solutions, we use the estimates for AgmonDouglis-Nirenberg systems and the homotopy argument. Moreover, our result can be quickly generalized 
to arbitrary $W_{p}^{k}(\Omega)$ Sobolev spaces. In order to do so, one would have to show $W_{p}^{1}(\Omega)$-estimates for solutions to (19) (see Sect. 4).

To prove Theorem 1 we will first consider the family of equations with homogeneous boundary conditions

$$
\begin{array}{ll}
-\nu \Delta \mathbf{u}+\tau \nabla \Pi=\mathbf{f} & \text { in } \Omega, \\
\tau \operatorname{div} \mathbf{u}+(1-\tau) \Pi=g & \text { in } \Omega, \\
\mathbf{u}_{\text {tan }}=\mathbf{0} & \text { on } \Gamma, \\
\Pi=\Pi_{\text {in,out }}^{k} & \text { on } \Gamma_{\text {in }, \text { out }}^{k}, \\
\mathbf{u} \cdot \mathbf{n}=0 & \text { on } \Gamma_{\text {lat }},
\end{array}
$$

where $\tau \in[0,1]$ and $\Pi_{i n, o u t}^{k}=0$. In Sect. 3 we will prove the following result:

Proposition 1.1. Let $1<p<\infty$ be fixed. Suppose that $\mathbf{f} \in L_{p}(\Omega), g \in W_{p}^{1}(\Omega)$ and $\left.\Pi_{\text {in,out }}^{k}\right|_{\Gamma_{\text {in out }}^{k}}=0$. Then, there exists a unique solution $(\mathbf{u}, \Pi) \in W_{p}^{2}(\Omega) \times W_{p}^{1}(\Omega)$ to $(4)$ such that

$$
\|\mathbf{u}\|_{W_{p}^{2}(\Omega)}+\|\Pi\|_{W_{p}^{1}(\Omega)} \leq c\left(\frac{1}{\nu}, \Omega\right)\left(\|\mathbf{f}\|_{L_{p}(\Omega)}+\|g\|_{W_{p}^{1}(\Omega)}\right) .
$$

The case when $\Pi_{i n, \text { out }}^{k} \neq 0$ will be discussed in Sect. 4 . There we also demonstrate the proof of Theorem 1. Finally, in Sect. 5 we show an application of the obtained results for the Navier-Stokes equations

$$
\begin{array}{ll}
\mathbf{v}, t+\operatorname{rot} \mathbf{v} \times \mathbf{v}+\nabla\left(p+\frac{1}{2}|\mathbf{v}|^{2}\right)-\nu \Delta \mathbf{v}=\mathbf{f} & \text { in } \Omega^{T}=\Omega \times(0, T), \\
\operatorname{div} \mathbf{v}=0 & \text { in } \Omega^{T}, \\
p+\frac{1}{2}|\mathbf{v}|^{2}=\Pi_{\text {in }, \text { out }}^{k} & \text { on } \Gamma_{\text {in, out }}^{k}, \\
\mathbf{v}_{\text {tan }}=\mathbf{0} & \text { on } \Gamma, \\
\mathbf{v} \cdot \mathbf{n}=0 & \text { on } \Gamma_{\text {lat }}, \\
\left.\mathbf{v}\right|_{t=0}=\mathbf{v}_{0} & \text { on } \Omega \times\{t=0\} .
\end{array}
$$

For the above system we have:

Theorem 2. Let Lip stand for lipschitz continuous. Suppose that $\Pi_{\text {in,out }}^{k} \in \operatorname{Lip}\left(0, T ; H^{\frac{1}{2}}\left(\Gamma_{\text {in,out }}^{k}\right)\right), \mathbf{f} \in$ $\operatorname{Lip}\left(0, T ; H^{1}(\Omega)\right), \mathbf{v}_{0} \in H^{1}(\Omega)$ and $\operatorname{div} \mathbf{v}_{0}=0$. Then:

- there exists an almost everywhere regular weak solution (see below) $\mathbf{v}(t)$ to $(5)$ on $[0, T]$. This solution is strong and unique for any $t \in\left[0, t_{0}\right]$, where $t_{0}$ is determined by $\left\|\mathbf{v}_{0}\right\|_{H^{1}(\Omega)}$.

- If $\left\|\mathbf{v}_{0}\right\|_{H^{1}(\Omega)}$ is small enough, then $\mathbf{v}(t)$ is strong and unique on $[0, T]$.

The definitions of weak, strong and almost everywhere regular solutions to (5) are in Sect. 5 (see Definitions 5.3, 5.2 and Lemma 5.4). As we observed after Theorem 1 one could generalize Theorem 2 to arbitrary Sobolev spaces by showing $W_{p}^{1}(\Omega)$-estimates for solutions to $(25)$.

A claim very similar to Theorem 2 (global existence of weak solutions and local-in-time existence of regular solutions) was suggested in [3, Sect. 6$]$ with a remark that its proof would not differ substantially from the case of Dirichlet conditions. Indeed, the main source of computational difficulties is the nonlinear term $(\mathbf{v} \cdot \nabla) \mathbf{v}$. When we test it with e.g. $\mathbf{v}$, it yields a boundary integral which so far can be only eliminated under additional assumptions on the magnitude of the initial data and certain norms of $\Pi_{\text {in out }}^{k}$ on $\Gamma_{\text {in,out }}^{k}$ (cf. [3, Theorem 6]). Problem (5) appears in [20, cf. (1.6) - (1.10)]. Under sligthly less restrictive assumptions on the regularity of the data and using the Schauder's fixed point theorem the short-time existence of regular solutions to (5) is shown (see Theorem 1.3). Subsequently, if in addition certain norms of $\mathbf{v}_{0}, \Gamma_{\text {in out }}^{k}$ and $\mathbf{f}$ are small, then the regular solution is global in time (see Theorem 5.3). This shows the main difference from our result: for obtaining global and regular solutions we only require $\left\|\mathbf{v}_{0}\right\|_{H^{1}(\Omega)}$ to be small. The case of (5) with non-vanishing tangential components of $\mathbf{v}$ on all parts of the boundary was formulated and also proved in [20] (see Theorem 5.4) and [21, Theorem 5.1]. For $k=2$ problem (5) was studied in e.g. [22,23]. 
Clearly, when we use the non-linear term in its conservative form, then the boundary integral does not appear. While, as pointed out in [3, Sect. 4], conditions $(5)_{3,4}$ are not fulfilled by Poiseuille flow with a constant total pressure, our pressure on in- and out-flow boundaries does not need to be constant and can therefore be chosen as those of Poiseuille flow, which means our solutions do indeed include Poiseuille flow. There is in any case much to be said in favor of prescribing the total pressure $\Pi=p+\frac{1}{2}|v|^{2}$, as in case of the flow of an ideal fluid the total pressure is conserved along flow lines in the absence of gravity, otherwise it is $\Pi+x_{3}$, if $-\mathbf{e}_{3}$ is the force of gravity. We are, of course, not discussing an ideal fluid here, but if the inflow on the boundary comes from a tank nearby with fairly slow flow, then the viscosity will not have a very large influence on the total pressure, and we may set it equal to the hydrostatic pressure on the inflow boundary as a reasonable approximation.

For further references (e.g. non-stationary Stokes system or stationary Navier-Stokes equations) we refer the reader to the Introduction in [24], where also problem (5) in $\Omega \subset \mathbb{R}^{2}$ was studied.

In the subsequent sections, to improve readability we will omit the index $k$ in case of $\Pi_{\text {in,out }}$ and $\Gamma_{\text {in }, \text { out }}$.

\section{Estimates}

In this section we discuss some estimates for solutions to (4) with $\Pi=0$ on $\Gamma_{\text {in,out }}$. We start with an observation about the boundary conditions for $\mathbf{u}$. From $(4)_{3,5}$ we infer that $\mathbf{u}=\mathbf{0}$ on $\Gamma_{\text {lat }}$. To find the condition on $\Gamma_{\text {in,out }}$ we recall that $(4)_{3}$ implies that (see e.g. p. 136 in [25])

$$
\left.\operatorname{div} \mathbf{u}\right|_{\Gamma}=\left.\frac{\partial u_{n}}{\partial \mathbf{n}}\right|_{\Gamma}
$$

thus (4) 2,3 give

$$
\begin{aligned}
& \mathbf{u}_{t a n}=\mathbf{0} \\
& \frac{\partial u_{n}}{\partial \mathbf{n}}=0 \quad \text { on } \Gamma_{\text {in }, \text { out }} .
\end{aligned}
$$

For $\tau \notin\{0,1\}$ we solve $(4)_{2}$ for $\Pi$

$$
\Pi=\frac{1}{1-\tau}(g-\tau \operatorname{div} \mathbf{u})
$$

and use it in $(4)_{1}$, thus

$$
-\nu \Delta \mathbf{u}-\frac{\tau^{2}}{1-\tau} \nabla \operatorname{div} \mathbf{u}=\mathbf{f}-\frac{\tau}{1-\tau} \nabla g
$$

Summarizing

$$
\begin{array}{ll}
-\nu \Delta \mathbf{u}-\frac{\tau^{2}}{1-\tau} \nabla \operatorname{div} \mathbf{u}=\mathbf{f}-\frac{\tau}{1-\tau} \nabla g & \text { in } \Omega, \\
\mathbf{u}=\mathbf{0} & \text { on } \Gamma_{\text {lat }}, \\
\mathbf{u}_{\text {tan }}=\mathbf{0} & \text { on } \Gamma_{\text {in }, \text { out }} \\
\frac{\partial u_{n}}{\partial \mathbf{n}}=0 & \text { on } \Gamma_{\text {in }, \text { out }}
\end{array}
$$

Lemma 2.1. Suppose that $1<p<\infty, \mathbf{f} \in L_{p}(\Omega)$ and $\nabla g \in L_{p}(\Omega)$. Then, any solution to (9) satisfies

$$
\|\mathbf{u}\|_{W_{p}^{2}(\Omega)} \leq c\left(\frac{1}{1-\tau}, \frac{1}{\nu}, \Omega\right)\left(\|\mathbf{f}\|_{L_{p}(\Omega)}+\|\nabla g\|_{L_{p}(\Omega)}\right) .
$$

Proof. To derive the estimates for the solution to (9) we introduce a partition of unity $\sum_{k=0}^{N} \zeta_{k}(\mathbf{x})=1$ on $\Omega$. Let $\mathbf{u}^{(k)}=\mathbf{u} \zeta_{k}$ and $\Omega^{(k)}=\Omega \cap \operatorname{supp} \zeta_{k}$. Then $\mathbf{u}^{(k)}$ satisfies

$$
\begin{aligned}
&-\nu \Delta \mathbf{u}^{(k)}-\frac{\tau^{2}}{1-\tau} \nabla \operatorname{div} \mathbf{u}^{(k)} \\
&=\mathbf{f}^{(k)}-\frac{\tau}{1-\tau} \nabla g \zeta_{k}-3 \nabla \mathbf{u} \cdot \nabla \zeta_{k}-\mathbf{u} \Delta \zeta_{k}-\operatorname{div} \mathbf{u} \nabla \zeta_{k}-\mathbf{u} \nabla^{2} \zeta_{k} \equiv \mathbf{F}^{(k)} \quad \text { in } \Omega^{(k)}
\end{aligned}
$$


with proper boundary conditions. It is clear that we can choose this partition of unity in such a way that exactly one of the following cases occurs:

1. $\operatorname{supp} \zeta_{k} \cap \Gamma=\emptyset$. This is the whole-space problem since $\mathbf{u}^{(k)}=\mathbf{0}$ on $\partial\left(\operatorname{supp} \zeta_{k} \cap \Omega\right)$. Without loss of generality we may further assume that $x_{3} \geq 0$, thereby reducing this case to half-space problem with zero Dirichlet boundary condition. Then, Theorem 14.1 [26] implies

$$
\left\|\mathbf{u}^{(k)}\right\|_{W_{p}^{2}\left(\Omega^{(k)}\right)} \leq c\left(\frac{1}{\nu}, \Omega\right)\left\|\mathbf{F}^{(k)}\right\|_{L_{p}\left(\Omega^{(k)}\right)}, \quad \text { for } 1<p<\infty .
$$

2. $\operatorname{supp} \zeta_{k} \cap \Gamma_{\text {lat }} \neq \emptyset$ but $\operatorname{supp} \zeta_{k} \cap \Gamma_{\text {in, out }}=\emptyset$. In this case we transform $\operatorname{supp} \zeta_{k} \cap \Omega$ into the half space and use Theorem 14.1 from [26] which eventually yields (10).

3. $\operatorname{supp} \zeta_{k} \cap \Gamma_{\text {lat }}=\emptyset$ but $\operatorname{supp} \zeta_{k} \cap \Gamma_{\text {in out }} \neq \emptyset$. Through rotations and translation we reduce this case to the half-space $x_{3} \geq 0$. Then $(9)_{4}$ implies that $\frac{\partial u_{3}^{(k)}}{\partial x_{3}}=u_{3} \zeta_{k, x_{3}}$. In order to make the right-hand side equal to zero, we may assume that e.g. $\zeta_{k}(\mathbf{x})=\psi\left(x_{1}, x_{2}\right) \phi\left(x_{3}\right)$, where $\phi\left(x_{3}\right)$ is constant and equals 1 in the neighborhood of $x_{3}=0$. Then $u_{3, x_{3}}^{(k)}=0$. From $(9)_{3}$ we instantly deduce that $u_{1}^{(k)}=u_{2}^{(k)}=0$. This observation suggests the following reflection

$$
\overline{\mathbf{u}}^{(k)}(\mathbf{x})= \begin{cases}\mathbf{u}^{(k)}(\mathbf{x}) & x_{3} \geq 0, \\ \left(-u_{1}^{(k)}(\overline{\mathbf{x}}),-u_{2}^{(k)}(\overline{\mathbf{x}}), u_{3}^{(k)}(\overline{\mathbf{x}})\right) & x_{3}<0,\end{cases}
$$

where $\overline{\mathbf{x}}=\left(x_{1}, x_{2},-x_{3}\right)$. For $\mathbf{u}^{(k)}$ we use again [26, Theorem 14.1] and therefore get (10).

4. $\operatorname{supp} \zeta_{k} \cap \Gamma_{\text {lat }} \neq \emptyset$ and $\operatorname{supp} \zeta_{k} \cap \Gamma_{\text {in,out }} \neq \emptyset$. Let $\mathbf{x}_{0} \in \Gamma_{\text {lat }} \cap \Gamma_{\text {in,out }}$. Then we introduce a local Cartesian coordinate system $\mathbf{y}=\mathbf{\Psi}(\mathbf{x})=\mathbf{C}\left(\mathbf{x}-\mathbf{x}_{0}\right), \mathbf{x} \in \operatorname{supp} \zeta_{k} \cap \Gamma_{\text {lat }}, \mathbf{C}$ is an orthogonal matrix, in such a way that the normal $\mathbf{n}$ to $\Gamma_{l a t}$ at $\mathbf{x}_{0}$ is parallel to $\mathbf{e}_{3}$ (cf. Fig. 2). Let $U_{k}=\boldsymbol{\Psi}\left(\operatorname{supp} \zeta_{k} \cap \Gamma_{\text {lat }}\right)$ be parametrized by the graph of $y_{3}=\psi\left(y_{1}, y_{2}\right), y_{1} \geq 0$, where we assume that $\psi(0,0)=0$. Then $\Gamma_{\text {in,out }}$ is contained in the plane $y_{1}=0, \mathbf{e}_{3}=\frac{\left(\psi_{y_{1}}(0,0), \psi_{y_{2}}(0,0),-1\right)}{\sqrt{1+\psi_{y_{1}}^{2}(0,0)+\psi_{y_{2}}^{2}(0,0)}}$ and $\mathbf{e}_{1} \cdot\left(\psi_{y_{1}}\left(0, y_{2}\right), \psi_{y_{2}}\left(0, y_{2}\right),-1\right)=0$, which implies that $\psi_{y_{1}}\left(0, y_{2}\right)=0$. Therefore we may reflect $U_{k}$ symmetrically across the plane $y_{1}=0$, i.e.

$$
\bar{U}_{k}= \begin{cases}\left(y_{1}, y_{2}, \psi\left(y_{1}, y_{2}\right)\right) & y_{1} \geq 0 \\ \left(-y_{1}, y_{2}, \psi\left(y_{1}, y_{2}\right)\right) & y_{1}<0\end{cases}
$$

Since $U_{k}$ is of class $\mathcal{C}^{2}$, so is $\bar{U}_{k}$ and now we proceed as in Case (2).

Summing over $k$ gives for any $p \in(1, \infty)$

$$
\|\mathbf{u}\|_{W_{p}^{2}(\Omega)} \leq c\left(\frac{1}{1-\tau}, \frac{1}{\nu}, \Omega\right)\left(\|\mathbf{u}\|_{W_{p}^{1}(\Omega)}+\|\mathbf{f}\|_{L_{p}(\Omega)}+\|\nabla g\|_{L_{p}(\Omega)}\right) .
$$

To eliminate $\|\mathbf{u}\|_{W_{p}^{1}(\Omega)}$ from the right-hand side we note that any weak solution $\mathbf{u}$ to (9) satisfies

$$
\nu\|\nabla \mathbf{u}\|_{L_{2}(\Omega)}^{2}+\frac{\tau^{2}}{1-\tau}\|\operatorname{div} \mathbf{u}\|_{L_{2}(\Omega)}^{2}=\int_{\Omega}\left(\mathbf{f}-\frac{\tau}{1-\tau} \nabla g\right) \cdot \mathbf{u} \mathrm{d} x,
$$

where the boundary integrals vanish due to $(9)_{2,3,4}$ and (6). Applying the Hölder and Cauchy inequalities to the right-hand side along with the Poincaré inequality (for $\mathbf{u}$ vanishing on a piece of $\partial \Omega$ see e.g. [27, Ch. 4, Sect. 4.5]) yields

$$
\|\mathbf{u}\|_{H^{1}(\Omega)}^{2} \leq c\left(\frac{1}{1-\tau}, \frac{1}{\nu}, \Omega\right)\left(\|\mathbf{f}\|_{L_{2}(\Omega)}^{2}+\|\nabla g\|_{L_{2}(\Omega)}^{2}\right) .
$$

Thus

$$
\|\mathbf{u}\|_{L_{2}(\Omega)} \leq c\left(\frac{1}{1-\tau}, \frac{1}{\nu}, \Omega\right)\left(\|\mathbf{f}\|_{L_{2}(\Omega)}+\|\nabla g\|_{L_{2}(\Omega)}\right) .
$$

To complete the proof we use the Gagliardo-Nirenberg inequality

$$
\|\mathrm{D} \mathbf{u}\|_{L_{p}(\Omega)} \leq c_{1}\left\|\mathrm{D}^{2} \mathbf{u}\right\|_{L_{p}(\Omega)}^{\alpha}\|\mathbf{u}\|_{L_{2}(\Omega)}^{1-\alpha}+c_{2}\|\mathbf{u}\|_{L_{2}(\Omega)},
$$




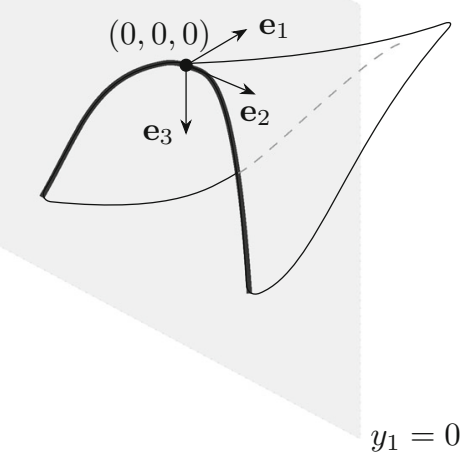

FIG. 2. $\Gamma_{\text {lat }} \cap \zeta_{k}$ as a graph of $y_{3}=\psi\left(y_{1}, y_{2}\right), y_{1} \geq 0$

which holds on Lipschitz domains provided that

$$
\frac{1}{p}=\frac{1}{3}+\left(\frac{1}{p}-\frac{2}{3}\right) \alpha+\frac{1-\alpha}{2} \quad \text { and } \quad \frac{1}{2} \leq \alpha \leq 1 .
$$

The latter condition is satisfied for $p \geq 2$. Finally, from the Poincaré and the Young inequalities

$$
\|\mathbf{u}\|_{W_{p}^{1}(\Omega)} \leq \epsilon\|\mathbf{u}\|_{W_{p}^{2}(\Omega)}+\frac{c}{\epsilon}\|\mathbf{u}\|_{L_{2}(\Omega)} .
$$

Taking $\epsilon>0$ small enough and using (11) completes the proof.

Remark 2.2. To derive (9) we assumed that $\tau \neq 0$. Since $\tau>0$ is arbitrary, by continuity Lemma 2.1 remains true when $\tau=0$. In this case we would have

$$
\|\mathbf{u}\|_{W_{p}^{2}(\Omega)} \leq c\left(\frac{1}{\nu}, \Omega\right)\|\mathbf{f}\|_{L_{p}(\Omega)} .
$$

Remark 2.3. From Lemma 2.1 and (8) it follows that

$$
\|\nabla \Pi\|_{L_{p}(\Omega)} \leq c\left(\frac{1}{1-\tau}, \frac{1}{\nu}, \Omega\right)\left(\|g\|_{W_{p}^{1}(\Omega)}+\tau\|\mathbf{u}\|_{W_{p}^{2}(\Omega)}\right), \quad 1<p<\infty .
$$

We easily observe that Lemma 2.1 does not work when $\tau=1$ in (4). Before we discuss this case, we establish the uniqueness of solutions to (4) for any $\tau \in[0,1]$.

Remark 2.4. If $(\mathbf{u}, \Pi)$ is a solution to (4) with $\Pi=0$ on $\Gamma_{\text {in,out }}$, then it is unique.

Indeed: assume that $\left(\mathbf{u}_{1}, \Pi_{1}\right)$ and $\left(\mathbf{u}_{2}, \Pi_{2}\right)$ solve (4). Then $\mathbf{U}=\mathbf{u}_{1}-\mathbf{u}_{2}$ and $\Psi=\Pi_{1}-\Pi_{2}$ solve

$$
\begin{array}{ll}
-\nu \Delta \mathbf{U}+\tau \nabla \Psi=\mathbf{0} & \text { in } \Omega, \\
\tau \operatorname{div} \mathbf{U}+(1-\tau) \Psi=0 & \text { in } \Omega, \\
\mathbf{U}_{\text {tan }}=\mathbf{0} & \text { on } \Gamma, \\
\Psi=0 & \text { on } \Gamma_{\text {in }, \text { out }}, \\
\mathbf{U} \cdot \mathbf{n}=0 & \text { on } \Gamma_{\text {lat }} .
\end{array}
$$


Next, we multiply $(12)_{1,2}$ by $\mathbf{U}$ and $\Psi$, respectively, and integrate over $\Omega$. We use that $-\Delta=\operatorname{rot}^{2}-\nabla \operatorname{div}$ and get

$$
\begin{aligned}
& \|\operatorname{rot} \mathbf{U}\|_{L_{2}(\Omega)}^{2}+\|\operatorname{div} \mathbf{U}\|_{L_{2}(\Omega)}^{2} \\
& \quad-\int_{\Gamma} \mathbf{U} \cdot \mathbf{n} \operatorname{div} \mathbf{U} \mathrm{d} S+\tau \int_{\Gamma} \mathbf{U} \cdot \mathbf{n} \Psi \mathrm{d} S-\tau \int_{\Omega} \Psi \operatorname{div} \mathbf{U} \mathrm{d} x=0
\end{aligned}
$$

and

$$
\tau \int_{\Omega} \operatorname{div} \mathbf{U} \Psi \mathrm{d} x+(1-\tau)\|\Psi\|_{L_{2}(\Omega)}^{2}=0 .
$$

Using $(12)_{5}$ and following the reasoning that led to $(7)$ we conclude that the first boundary integral vanishes. The second one vanishes due to $(12)_{5,6}$. Adding the above equalities we obtain

$$
\|\operatorname{rot} \mathbf{U}\|_{L_{2}(\Omega)}^{2}+\|\operatorname{div} \mathbf{U}\|_{L_{2}(\Omega)}^{2}+(1-\tau)\|\Psi\|_{L_{2}(\Omega)}^{2}=0 .
$$

If $\tau \in[0,1)$ we see that $\mathbf{U}=\mathbf{0}$ and $\Psi=0$. For $\tau=1$ function $\Psi=0$ as it equals zero on $\Gamma_{\text {in,out }}$.

Now we can analyze (4) when $\tau=1$.

Lemma 2.5. Suppose that $\mathbf{f} \in L_{p}(\Omega)$ and $g \in W_{p}^{1}(\Omega)$. Then any solution to (4) with $\tau=1$ satisfies

$$
\|\mathbf{u}\|_{W_{p}^{2}(\Omega)}+\|\Pi\|_{W_{p}^{1}(\Omega)} \leq c\left(\frac{1}{\nu}, \Omega\right)\left(\|\mathbf{f}\|_{L_{p}(\Omega)}+\|g\|_{W_{p}^{1}(\Omega)}\right) .
$$

Proof. As in the proof of Lemma 2.1 we introduce a partition of unity $\sum_{k=0}^{N} \zeta_{k}(\mathbf{x})=1$ on $\Omega$ and we write

$$
\begin{aligned}
\mathbf{u}^{(k)} & =\mathbf{u} \zeta_{k}, \\
\Pi^{(k)} & =\Pi \zeta_{k}, \\
\Omega^{(k)} & =\Omega \cap \operatorname{supp} \zeta_{k} .
\end{aligned}
$$

Multiplying $(4)_{1,2}$ by $\zeta_{k}$ yields

$$
\begin{aligned}
-\nu \Delta \mathbf{u}^{(k)}+\nabla \Pi^{(k)} & =\mathbf{f}^{(k)}-2 \nabla \mathbf{u} \cdot \nabla \zeta_{k}-\mathbf{u} \nabla^{2} \zeta_{k}+\Pi \nabla \zeta_{k} \equiv \mathbf{F}^{(k)}, \\
\operatorname{div} \mathbf{u}^{(k)} & =g^{(k)}+\mathbf{u} \cdot \nabla \zeta_{k} \equiv G^{(k)} .
\end{aligned}
$$

When $k$ is fixed, four cases are possible. There are identical to the cases considered in the proof of Lemma 2.1. However, in cases (3) and (4) we need to define the reflection of the pressure through $\Gamma_{\text {in,out }}$. Since $\left.\Pi\right|_{\Gamma_{\text {in }, \text { out }}}=0$, we set

$$
\bar{\Pi}(\overline{\mathbf{x}})= \begin{cases}\Pi(\mathbf{x}), & x_{3} \geq 0, \\ -\Pi(\overline{\mathbf{x}}), & x_{3}<0,\end{cases}
$$

where $\overline{\mathbf{x}}=\left(x_{1}, x_{2},-x_{3}\right)$. For (13) in the whole space we have the following estimate (cf. [28, Theorem IV.2.1])

$$
\left\|\mathbf{u}^{(k)}\right\|_{W_{p}^{2}\left(\Omega^{(k)}\right)}+\left\|\Pi^{(k)}\right\|_{W_{p}^{1}\left(\Omega^{(k)}\right)} \leq c(\Omega)\left(\left\|\mathbf{F}^{(k)}\right\|_{L_{p}\left(\Omega^{(k)}\right)}+\left\|G^{(k)}\right\|_{W_{p}^{1}\left(\Omega^{(k)}\right)}\right) .
$$

By [28, Theorem IV.3.2] we obtain the identical estimate for the half-space case. Summing over $k$ yields

$$
\begin{aligned}
& \|\mathbf{u}\|_{W_{p}^{2}(\Omega)}+\|\Pi\|_{W_{p}^{1}(\Omega)} \\
& \quad \leq c\left(\frac{1}{\nu}, \Omega\right)\left(\|\mathbf{f}\|_{L_{p}(\Omega)}+\|g\|_{W_{p}^{1}(\Omega)}+\|\mathbf{u}\|_{W_{p}^{1}(\Omega)}+\|\Pi\|_{L_{p}(\Omega)}\right) .
\end{aligned}
$$

To get rid of $\mathbf{u}$ and $p$ from the right-hand side we prove that

$$
\|\mathbf{f}\|_{L_{p}(\Omega)}+\|g\|_{W_{p}^{1}(\Omega)} \geq c\left(\frac{1}{\nu}, \Omega\right)\left(\|\mathbf{u}\|_{W_{p}^{1}(\Omega)}+\|\Pi\|_{L_{p}(\Omega)}\right) .
$$


Suppose that the above inequality is not true. Then, there exists a sequence $\left(\mathbf{u}_{m}, \Pi_{m}, \mathbf{f}_{m}, g_{m}\right) \in W_{p}^{2}(\Omega) \times$ $W_{p}^{1}(\Omega) \times L_{p}(\Omega) \times W_{p}^{1}(\Omega)$ such that they solve (4) with $\tau=1, \Pi_{m}=0$ on $\Gamma_{\text {in,out }}$ and

$$
m\left(\left\|\mathbf{f}_{m}\right\|_{L_{p}(\Omega)}+\left\|g_{m}\right\|_{W_{p}^{1}(\Omega)}\right) \leq\left\|\mathbf{u}_{m}\right\|_{W_{p}^{1}(\Omega)}+\left\|\Pi_{m}\right\|_{L_{p}(\Omega)} .
$$

As system (4) is linear, and the above inequality is homogeneous, the function vector

$$
\left(\mathbf{v}_{m}, \Psi_{m}, \hat{\mathbf{f}}_{m}, \hat{g}_{m}\right)=\frac{1}{\left\|\mathbf{u}_{m}\right\|_{W_{p}^{1}(\Omega)}+\left\|\Pi_{m}\right\|_{L_{p}(\Omega)}}\left(\mathbf{u}_{m}, \Pi_{m}, \mathbf{f}_{m}, g_{m}\right)
$$

solves the same system and fulfills the same inequality. Clearly

$$
\left\|\mathbf{v}_{m}\right\|_{W_{p}^{1}(\Omega)}+\left\|\Psi_{m}\right\|_{L_{p}(\Omega)}=1
$$

therefore

$$
m\left(\left\|\hat{\mathbf{f}}_{m}\right\|_{L_{p}(\Omega)}+\left\|\hat{g}_{m}\right\|_{W_{p}^{1}(\Omega)}\right) \leq\left\|\mathbf{v}_{m}\right\|_{W_{p}^{1}(\Omega)}+\left\|\Psi_{m}\right\|_{L_{p}(\Omega)}=1,
$$

thus

$$
\left\|\hat{\mathbf{f}}_{m}\right\|_{L_{p}(\Omega)}+\left\|\hat{g}_{m}\right\|_{W_{p}^{1}(\Omega)} \leq \frac{1}{m}
$$

and

$$
\left\|\mathbf{v}_{m}\right\|_{W_{p}^{2}(\Omega)}+\left\|\Psi_{m}\right\|_{W_{p}^{1}(\Omega)} \leq c\left(1+\frac{1}{m}\right) .
$$

After selecting a subsequence, which we denote by the same symbols as the original one, we have that this subsequence converges weakly to a $(\mathbf{v}, \Psi, \mathbf{0}, 0) \in W_{p}^{2}(\Omega) \times W_{p}^{1}(\Omega) \times L_{p}(\Omega) \times W_{p}^{1}(\Omega)$. As $\mathbf{v}_{m} \rightarrow \mathbf{v}$ in $W_{p}^{1}(\Omega)$ we have

$$
0 \leftarrow \hat{g}_{m}=\operatorname{div} \mathbf{v}_{m} \rightarrow \operatorname{div} \mathbf{v} \quad \text { in } L_{p}(\Omega)
$$

and with $\hat{\mathbf{f}}_{m}=-\nu \Delta \mathbf{v}_{m}+\nabla \Psi_{m}$ we have

$$
\hat{\mathbf{f}}_{m} \rightarrow \mathbf{0} \quad \text { and } \quad-\nu \Delta \mathbf{v}_{m}+\nabla \Psi_{m} \rightarrow-\nu \Delta \mathbf{v}+\nabla \Psi \quad \text { in } L_{p}(\Omega),
$$

therefore $-\nu \Delta \mathbf{v}+\nabla \Psi=\mathbf{0}$. Thus, $(\mathbf{v}, \Psi, \mathbf{0}, 0)$ solves (4) with $\tau=1$ as well. By the uniqueness of the solutions to (4) (see Remark 2.4) we have $(\mathbf{v}, \Psi, \mathbf{0}, 0)=\mathbf{0}$, while by the Relich-Kondrachov theorem this contradicts (14).

\section{Existence}

The existence of solutions to (1) is obtained in two steps. First, we look into (4) and use homotopy argument. Next, we consider the non-homogeneous problem (see Sect. 4).

Let us define the family of operators $L_{\tau}: B_{1} \rightarrow B_{2}$, where

$$
L_{\tau}\left[\begin{array}{c}
\mathbf{u} \\
\Pi
\end{array}\right]=\left[\begin{array}{c}
-\nu \Delta \mathbf{u}+\tau \nabla \Pi \\
\tau \operatorname{div}(\mathbf{u})+(1-\tau) \Pi
\end{array}\right], \quad \tau \in[0,1]
$$

and

$$
\begin{aligned}
& B_{1}=\left\{\left[\begin{array}{l}
\mathbf{u} \\
\Pi
\end{array}\right]:(\mathbf{u}, \Pi) \in W_{p}^{2}(\Omega) \times W_{p}^{1}(\Omega),\left.\mathbf{u}_{\text {tan }}\right|_{\Gamma}=\mathbf{0},\left.\mathbf{u} \cdot \mathbf{n}\right|_{\Gamma_{\text {lat }}}=0,\left.\Pi\right|_{\Gamma_{\text {in }, \text { out }}}=0\right\}, \\
& B_{2}=\left\{\left[\begin{array}{l}
\mathbf{f} \\
g
\end{array}\right]: \mathbf{f} \in L_{p}(\Omega), g \in W_{p}^{1}(\Omega), \int_{\Omega} g \mathrm{~d} \mathbf{x}=0\right\} .
\end{aligned}
$$

By $\|\cdot\|_{B_{1}}$ and $\|\cdot\|_{B_{2}}$ we denote the norms on $B_{1}$ and $B_{2}$ which are induced by the standard scalar products, respectively.

It is easy to check that $L_{\tau}$ is injective. 
Lemma 3.1. Let $B_{1}$ and $B_{2}$ be two Banach spaces and assume $L_{\tau}: B_{1} \rightarrow B_{2}, \tau \in[0,1]$ is a family of continuous injective linear mappings from $B_{1}$ to $B_{2}$ such that

$$
\left\|L_{\tau_{1}}-L_{\tau_{2}}\right\|_{L\left(B_{1}, B_{2}\right)} \leq c_{1}\left|\tau_{1}-\tau_{2}\right| \quad \forall_{\tau_{1}, \tau_{2} \in[0,1]} .
$$

In addition assume there is a function $\gamma:[0,1] \rightarrow \mathbb{R}_{+}$such that

$$
\|v\|_{B_{1}} \leq \gamma(\tau)\left\|L_{\tau} v\right\|_{B_{2}} \text {. }
$$

Then, there exists a constant $c_{2}$ such that

$$
\|v\|_{B_{1}} \leq c_{2}\left\|L_{\tau} v\right\|_{B_{2}}
$$

and if $L_{0}$ is surjective then $L_{1}$ is also surjective.

Proof. Let $\tau_{0} \in[0,1]$ be fixed. By the injectivity of $L_{\tau}$ and (16) the expression

$$
\sup _{v \neq 0} \frac{\|v\|_{B_{1}}}{\left\|L_{\tau} v\right\|_{B_{2}}}
$$

is finite for all $\tau \in[0,1]$ and we could replace the original $\gamma$ by it without invalidating (16). Let us assume that $\gamma$ now equals that supremum. Then for $\tau_{1}, \tau_{2} \in[0,1]$ we have

$$
\begin{aligned}
& \|v\|_{B_{1}} \leq \gamma\left(\tau_{1}\right)\left\|L_{\tau_{1}} v\right\|_{B_{2}} \leq \gamma\left(\tau_{1}\right)\left(\left\|L_{\tau_{2}} v\right\|_{B_{2}}+\left\|L_{\tau_{2}} v-L_{\tau_{1}} v\right\|_{B_{2}}\right) \\
& \quad \leq \gamma\left(\tau_{1}\right)\left(\left\|L_{\tau_{2}} v\right\|_{B_{2}}+c_{1}\left|\tau_{1}-\tau_{2}\right|\|B\|_{B_{1}}\right)
\end{aligned}
$$

thus

$$
\left(1-c_{1} \gamma\left(\tau_{1}\right)\left|\tau_{1}-\tau_{2}\right|\right)\|v\|_{B_{1}} \leq \gamma\left(\tau_{1}\right)\left\|L_{\tau_{2}} v\right\|_{B_{2}} .
$$

If $c_{1} \gamma\left(\tau_{1}\right)\left|\tau_{1}-\tau_{2}\right|<1$ we obtain

$$
\gamma\left(\tau_{2}\right) \leq \frac{\gamma\left(\tau_{1}\right)}{1-c_{1} \gamma\left(\tau_{1}\right)\left|\tau_{1}-\tau_{2}\right|} .
$$

As $\tau_{1}$ and $\tau_{2}$ are interchangeable, this implies $\gamma$ is continuous and therefore has a finite maximum on $[0,1]$.

Let now $L_{\tau_{0}}$ be surjective. Then $L_{\tau_{0}} v=g$ is equivalent with

$$
L_{\tau} v=g+L_{\tau} v-L_{\tau_{0}} v .
$$

We define $F: B_{1} \rightarrow B_{1}$ by

$$
F(v)=L_{\tau}^{-1}\left(g+L_{\tau} v-L_{\tau_{0}} v\right) .
$$

Then, $L_{\tau}$ is surjective if and only if $F$ has a fixed point. By the properties of $L_{\tau}$

$$
\|F(u)-F(v)\|_{B_{1}} \leq c_{2}\left\|L_{\tau}(u-v)-L_{\tau_{0}}(u-v)\right\| \leq c_{2} c_{1}\left|\tau-\tau_{0}\right|\|u-v\| .
$$

If we take $\tau$ close enough to $\tau_{0}$, then $c_{1} c_{2}\left|\tau-\tau_{0}\right|<1$ and by the Banach Fixed Point Theorem we conclude the surjectivity of $L_{\tau}$. To complete the proof we start with $\tau_{0}=0$ and go to 1 in a finite number of steps as $c_{1}$ and $c_{2}$ do not depend on $\tau$.

Remark 3.2. In the above proof we start with $\tau_{0}=0$ which corresponds to the following problem

$$
\begin{array}{ll}
-\nu \Delta \mathbf{u}=\mathbf{f} & \text { in } \Omega, \\
\mathbf{u}=\mathbf{0} & \text { on } \Gamma_{\text {lat }}, \\
\mathbf{u}_{\text {tan }}=\mathbf{0} & \text { on } \Gamma_{\text {in,out }}, \\
\frac{\partial u_{n}}{\partial \mathbf{n}}=0 & \text { on } \Gamma_{\text {in,out }} .
\end{array}
$$

The existence and regularity of solutions (cf. Remark 2.2) follow now from reflection and classical theory. Proof of Proposition 1.1. The proof follows immediately from Lemmas 2.5 and 3.1. 


\section{General Case}

Suppose now that $\left.\Pi\right|_{\Gamma_{\text {in out }}}=\Pi_{\text {in,out }}$. We introduce an auxiliary function $p: \Omega \rightarrow \mathbb{R}$ such that

$$
\begin{array}{ll}
-\Delta p=0 & \text { in } \Omega, \\
\frac{\partial p}{\partial \mathbf{n}}=0 & \text { on } \Gamma_{\text {lat }}, \\
p=\Pi_{\text {in,out }} & \text { on } \Gamma_{\text {in,out }} .
\end{array}
$$

One can easily show the existence of weak solutions to the above problem. These solutions satisfy

$$
\|p\|_{H^{1}(\Omega)} \leq c\left\|\Pi_{\text {in }, \text { out }}\right\|_{H^{\frac{1}{2}}\left(\Gamma_{\text {in }, \text { out }}\right)} .
$$

Then, the pair $(\mathbf{u}, \psi)$, where $\psi=\Pi-p$, solves

$$
\begin{array}{ll}
-\nu \Delta \mathbf{u}+\nabla \psi=\mathbf{f} & \text { in } \Omega, \\
\operatorname{div} \mathbf{u}=g & \text { in } \Omega, \\
\mathbf{u}_{\text {tan }}=\mathbf{0} & \text { on } \Gamma, \\
\psi=0 & \text { on } \Gamma_{\text {in }, \text { out }}, \\
\mathbf{u} \cdot \mathbf{n}=0 & \text { on } \Gamma_{\text {lat }} .
\end{array}
$$

From Proposition 1.1 we get the existence of solution $(\mathbf{u}, \psi)$ and the estimate

$$
\|\mathbf{u}\|_{H^{2}(\Omega)}+\|\psi\|_{H^{1}(\Omega)} \leq c\left(\frac{1}{\nu}, \Omega\right)\left(\|\mathbf{f}\|_{L_{2}(\Omega)}+\|g\|_{H^{1}(\Omega)}\right) .
$$

From (20), (21) and the existence of $\mathbf{u}, \psi$ and $p$ we get Theorem 1 .

\section{Navier-Stokes Equations}

As an application of the theory we have presented so far, we will consider the Navier-Stokes equations. Let us denote $\Pi=p+\frac{1}{2}|\mathbf{v}|^{2}$ and $\boldsymbol{\omega}=\operatorname{rot} \mathbf{v}$. We first consider the case when $\Pi=0$ on $\Gamma_{\text {in,out }}$. For a similar problem see e.g. $[29,30]$. Let us define

$$
\mathcal{H}=\left\{\mathbf{u} \in L_{2}(\Omega): \int_{\Omega} \mathbf{u} \cdot \nabla \psi \mathrm{d} x=0 \forall_{\psi \in H^{1}(\Omega)},\left.\psi\right|_{\partial \Gamma_{\text {in }, \text { out }}}=0\right\} .
$$

By $\|\cdot\|_{\mathcal{H}}$ we denote the norm on $\mathcal{H}$ induced by the standard scalar product $(\cdot, \cdot)$ on $\mathcal{H}$.

Let $\mathcal{P}: L_{2}(\Omega) \rightarrow \mathcal{H}$ be the orthogonal projector. Applying it to (5) we get

$$
\mathbf{v}, t+\nu A \mathbf{v}=-\mathcal{P}(\boldsymbol{\omega} \times \mathbf{v})+\mathcal{P} \mathbf{f}=: F(\mathbf{v}, t),
$$

where $A$ stands for the Stokes operator whose domain of the definition in this case is

$$
D(A)=\left\{\mathbf{u} \in H^{2} \cap \mathcal{H}:\left.\mathbf{u}_{\text {tan }}\right|_{\Gamma}=0\right\} .
$$

From Proposition 1.1 it follows that $A$ is positive self-adjoint with compact inverse. For such operators we have extensive theory (see e.g. Ch. 6 in [31] and the references in Comments on Chapter 6). In particular, in light of $[32$, Ch. 5] we have:

Definition 5.1. Assume that $H$ is a Hilbert space with the scalar product $(\cdot, \cdot)$ and $D(A) \subset H$ is a linear subspace. Suppose that $A: D(A) \rightarrow H$ is self-adjoint operator with a compact inverse. Let $u_{1}, u_{2}, \ldots \in$ $D(A)$ be a complete orthonormal system of eigenelements of $A$ with real eigenvalues $0<\lambda_{1} \leq \lambda_{2} \leq \cdots$. and

$$
D(A)=\left\{u \in H: \sum_{k=1}^{\infty} \lambda_{k}^{2}\left|\left(u, u_{k}\right)\right|^{2}<\infty\right\} .
$$


Assume that $f:\left[\lambda_{1}, \infty\right) \rightarrow \mathbb{R}$. Then we set

$$
D(f(A))=\left\{u \in H: \sum_{k=1}^{\infty}\left(f\left(\lambda_{k}\right)\right)^{2}\left|\left(u, u_{k}\right)\right|^{2}<\infty\right\}
$$

and define

$$
f(A) u=\sum_{k=1}^{\infty} f\left(\lambda_{k}\right)\left(u, u_{k}\right) u_{k}
$$

for $u \in D(f(A))$.

From the above definition it follows that for $\lambda_{1}>0$ we can define for any $s \geq 0$ and $u \in H$

$$
A^{s} u=\sum_{j=1}^{\infty} \lambda_{j}^{s}\left(u, u_{j}\right) u_{j}
$$

provided that the series $\sum_{j=1}^{\infty} \lambda_{j}^{2 s}\left|\left(u, u_{j}\right)\right|^{2}$ converges. For fractional powers of $A$ we have (see e.g. [33, Sect. 1.3.3.])

$$
\|u\|_{H^{2 s}(\Omega)} \leq c\left\|A^{s} u\right\|_{L_{2}(\Omega)}, \quad s \in[0,1] .
$$

Utilizing the Sobolev embeddings (see e.g. [33, Prop. 1.2.1]) we get

$$
\begin{aligned}
\|u\|_{L_{p}(\Omega)} & \leq c\|u\|_{H^{2 s}(\Omega)}, & & s \geq \frac{3 p-6}{4 p}, \\
\|u\|_{W_{p}^{1}(\Omega)} & \leq c\|u\|_{H^{2 s}(\Omega)}, & s & \geq \frac{5 p-6}{4 p} .
\end{aligned}
$$

Below we show the existence of weak and strong solutions to (5) by analyzing (22). These solutions are defined in Lemma 5.4 and Definition 5.2, respectively. We will use an abstract method from [34], which is an enhanced version of the theorem from [35, Sect. 1.1]. This method is based on the direct application of the next Lemma to (22). First, we need to define almost everywhere regular solutions.

Definition 5.2. We call a function $\mathbf{v}:\left(t, t^{\prime}\right) \rightarrow \mathcal{H}$ a strong solution to $(22)$ on the interval $\left(t, t^{\prime}\right)$ if $\mathbf{v}$ fulfills $(22)$ and $\mathbf{v} \in \mathcal{C}^{1}\left(\left(t, t^{\prime}\right), \mathcal{H}\right) \cap \mathcal{C}\left(\left(t, t^{\prime}\right), D(A)\right)$.

Definition 5.3. A solution $\mathbf{u}$ is called almost everywhere regular in a time interval $[0, T]$ if there is a countable collection of disjoint open subintervals $I_{k}(k \in S)$ such that $[0, T] \backslash\left(\bigcup_{k \in S} I_{k}\right)$ is a set of one-dimensional Lebesgue measure zero (see $[34,35]$ ) and $\mathbf{u}$ is strong on $I_{k}$ for all $k \in S$.

Lemma 5.4. (cf. Theorem in [34]) Let $T<+\infty$. Suppose that $A: D(A) \rightarrow \mathcal{H}$ is positive self-adjoint with compact inverse. Assume that there exists a fixed number $\mu \in(0,1)$ and a continuous increasing function $g: \mathbb{R}_{+} \rightarrow \mathbb{R}_{+}$such that the non-linearity $F: D\left(A^{\mu}\right) \times(0, T) \rightarrow H$ has the following properties:

$\left(\mathbf{C}_{1}\right)$ There exists a constant $c_{1}$ such that

$$
\|F(\mathbf{u}, t)\|_{\mathcal{H}} \leq g\left(\left\|A^{\frac{\mu}{2}} \mathbf{u}\right\|_{\mathcal{H}}\right)\left(\left\|A^{\mu} \mathbf{u}\right\|_{\mathcal{H}}+1\right)
$$

$\left(\mathbf{C}_{2}\right)$ For $\mathbf{u}, \mathbf{u}^{\prime} \in D\left(A^{\mu}\right), t, t^{\prime} \in(0, T)$ we have

$$
\begin{aligned}
& \left\|F(\mathbf{u}, t)-F\left(\mathbf{u}^{\prime}, t^{\prime}\right)\right\|_{\mathcal{H}} \\
& \quad \leq g\left(\left\|A^{\mu} \mathbf{u}\right\|_{\mathcal{H}}+\left\|A^{\mu} \mathbf{u}^{\prime}\right\|_{\mathcal{H}}\right)\left(\left\|A^{\mu}\left(\mathbf{u}-\mathbf{u}^{\prime}\right)\right\|_{\mathcal{H}}+\left|t-t^{\prime}\right|\right)
\end{aligned}
$$

and

$\left(\mathbf{C}_{3}\right)$ We have

$$
\|F(\mathbf{u}, t)\|_{\mathcal{H}} \leq g\left(\left\|A^{\frac{\mu}{2}} \mathbf{u}\right\|_{\mathcal{H}}\right)\left(\left\|A^{\mu} \mathbf{u}\right\|_{\mathcal{H}}+1\right)
$$

$$
\left\|A^{-1} F(\mathbf{u}, t)\right\|_{\mathcal{H}} \leq g\left(\|\mathbf{u}\|_{\mathcal{H}}\right)\left(\left\|A^{\frac{1}{2}} \mathbf{u}\right\|_{\mathcal{H}}^{2 \mu}+1\right) \quad \forall(\mathbf{u}, t) \in\left(D\left(A^{\mu}\right) \times(0, T)\right) .
$$


Then, for every $\mathbf{v}_{0} \in \mathcal{H}$ there exists a function $\mathbf{v}:[0, T] \rightarrow \mathcal{H}$ to (22) fulfilling the energy inequality

$$
\|\mathbf{v}(t)\|_{\mathcal{H}}^{2}+\nu \int_{0}^{t}\left\|A^{\frac{1}{2}} \mathbf{v}(s)\right\|_{\mathcal{H}}^{2} \mathrm{~d} s \leq c\left(\left\|\mathbf{v}_{0}\right\|_{\mathcal{H}}^{2}+\|\mathbf{f}\|_{L_{2}\left(0, T ; L_{2}(\Omega)\right)}^{2}\right)
$$

for all $t \leq T$. In addition, this function satisfies:

1. $A^{-1} \mathbf{v}(t) \in \mathcal{C}([0, t] ; \mathcal{H})$ and $\mathbf{v}(0)=\mathbf{v}_{0}$.

2. There exists a relatively open set $\mathcal{D} \subset[0, t]$ such that the Lebesgue measure of $[0, t] \backslash \mathcal{D}$ is zero and $\mathbf{v} \in \mathcal{C}^{1}(\mathcal{D} ; \mathcal{H}) \cap \mathcal{C}(\mathcal{D}, D(A))$, and

$$
\mathbf{v}_{t}+\nu A \mathbf{v}=F(\mathbf{v}, t) \quad \text { for } t \in \mathcal{D} .
$$

We also have:

- if $\mathbf{v}_{0} \in D(A)$, then $0 \in \mathcal{D}$,

- $\left\|A^{\frac{\mu}{2}} \mathbf{v}(t)\right\|_{\mathcal{H}} \rightarrow \infty$ as $t$ approaches the endpoints of $\mathcal{D} \backslash\{0, t\}$.

3. Function $\mathbf{v}$ is a weak solution to $(22)$, i.e. for any $\mathbf{u} \in \mathcal{C}^{1}([0, T], \mathcal{H}) \cap \mathcal{C}([0, T], D(A))$ we have

$$
\int_{\mathcal{D} \cap\left(t, t^{\prime}\right)}-(\mathbf{v}, A \mathbf{u})+(F(\mathbf{v}, t), \mathbf{u})+\left(\mathbf{v}, \mathbf{u}_{t}\right) \mathrm{d} \tau=\left(\mathbf{v}\left(t^{\prime}\right), \mathbf{u}\left(t^{\prime}\right)\right)-(\mathbf{v}(t), \mathbf{u}(t))
$$

for any $0 \leq t \leq t^{\prime} \leq T$.

4. If $\|\mathbf{v}(\tau)\|_{\mathcal{H}} \leq \epsilon \leq \epsilon_{0}$ for some $\epsilon_{0}>0$ on $\left[t_{1}, t_{2}\right] \subset[0, t]$, then $\left[t_{1}+\epsilon^{2}, t_{2}\right] \subset \mathcal{D}$. Also for all $\tau \in[0, t]$ we have

$$
\begin{array}{ll}
\|\mathbf{v}(\tau)\|_{\mathcal{H}}^{2} \leq \exp (2 \alpha \tau)\left\|\mathbf{v}_{0}\right\|_{\mathcal{H}}^{2}+C_{1} \frac{\exp (2 \alpha \tau)-1}{\alpha} \alpha \neq 0, \\
\|\mathbf{v}(\tau)\|_{\mathcal{H}}^{2} \leq\left\|\mathbf{v}_{0}\right\|^{2}+2 C_{1} \tau & \alpha=0,
\end{array}
$$

where $\alpha=C_{1}-\lambda_{1}$, with $\lambda_{1}$ being the lowest eigenvalue of $A$.

Therefore:

$\left(\mathbf{A}_{1}\right)$ If $C_{1}$ is sufficiently small, then the solution $\mathbf{v}(t)$ will become strong after a time determined by $C_{1}$ and $\mathbf{v}_{0}$.

$\left(\mathbf{A}_{2}\right)$ If both $C_{1}$ and $\left\|A \mathbf{v}_{0}\right\|_{\mathcal{H}}$ are small enough, then the solution $\mathbf{v}(t)$ is strong on the whole interval.

$\left(\mathbf{A}_{3}\right)$ Also, if for a $t_{0} \in \mathcal{D}$ there is a strong solution $\mathbf{u}(t)$ to $(22)$ on $\left[t_{0}, t\right]$ and $\mathbf{u}\left(t_{0}\right)=\mathbf{v}\left(t_{0}\right)$, then $\mathbf{u}(\tau)=\mathbf{v}(\tau)$ for $\tau \geq t_{0}$.

We easily see that assertions $(1)+(2)+(3)$ ensure that a weak solution $\mathbf{v}$ to $(22)$ is an almost everywhere weak regular solution. From the first part of assertion (4) it follows that if a weak solution is small enough, than it becomes the strong solution. The smallness of a weak solutions is guaranteed when the constant $C_{1}$ is small enough (see assertion (A1): indeed, if $C_{1} \leq \frac{\lambda_{1}}{2}$, then $\alpha \leq-\frac{\lambda_{1}}{2}$ and $(24)_{1}$ implies that for sufficiently large $t$

$$
\|\mathbf{v}(t)\|_{\mathcal{H}} \leq 2 \sqrt{\lambda_{1}^{-1} C_{1}} .
$$

If $C_{1}$ is small enough, we have that $\|\mathbf{v}(t)\|_{\mathcal{H}} \leq \epsilon_{0}$.

To use the above Lemma, let [cf. (22)]

$$
F(\mathbf{v}, t)=F_{1}(\mathbf{v}, t)+F_{2}(t) .
$$

Lemma 5.5. Suppose that $\mathbf{f} \in \operatorname{Lip}\left(0, T ; L_{2}(\Omega)\right), \mathbf{v}_{0} \in D(A)$. Then, there exists an almost everywhere regular weak solution $\mathbf{v}(t)$ to (22) on $[0, T]$. In addition, if $A \mathbf{v}_{0} \in \mathcal{H}$ is small, then the solution $\mathbf{v}(t)$ is strong on $[0, T]$. If $A \mathbf{v}_{0} \in \mathcal{H}$ is arbitrary, then $\mathbf{v}(t)$ remains strong for $t \in\left[0, t_{0}\right]$, where $t_{0}$ is determined by $\left\|A \mathbf{v}_{0}\right\|_{\mathcal{H}}$.

Proof. To prove this Lemma we use Lemma 5.4. We need to check the conditions $\left(\mathbf{C}_{1}\right),\left(\mathbf{C}_{2}\right)$ and $\left(\mathbf{C}_{3}\right)$.

For $\left(\mathbf{C}_{1}\right)$ we have

$$
(-\mathcal{P}(\boldsymbol{\omega} \times \mathbf{v})+\mathcal{P} \mathbf{f}, \mathbf{v})=\int_{\Omega} \boldsymbol{\omega} \times \mathbf{v} \cdot \mathcal{P} \mathbf{v} \mathrm{d} x+\int_{\Omega} \mathbf{f} \cdot \mathcal{P} \mathbf{v} \mathrm{d} x \leq\|\mathbf{f}\|_{L_{2}(\Omega)}\|\mathbf{v}\|_{\mathcal{H}}
$$


For the condition $\left(\mathbf{C}_{2}\right)$ we first note

$$
\left\|F(\mathbf{v}, t)-F\left(\mathbf{v}^{\prime}, t^{\prime}\right)\right\|_{\mathcal{H}} \leq\left\|F_{1}(\mathbf{v}, t)-F_{1}\left(\mathbf{v}^{\prime}, t^{\prime}\right)\right\|_{\mathcal{H}}+\left\|F_{2}(t)-F_{2}\left(t^{\prime}\right)\right\|_{\mathcal{H}} .
$$

Now we have

$$
\begin{aligned}
& \left\|F_{1}(\mathbf{v}, t)-F_{1}\left(\mathbf{v}^{\prime}, t^{\prime}\right)\right\|_{\mathcal{H}}=\left\|-\mathcal{P}\left(\boldsymbol{\omega} \times \mathbf{v}-\boldsymbol{\omega}^{\prime} \times \mathbf{v}^{\prime}\right)\right\|_{\mathcal{H}} \\
& \quad \leq\left\|\boldsymbol{\omega} \times \mathbf{v}-\boldsymbol{\omega}^{\prime} \times \mathbf{v}^{\prime}\right\|_{L_{2}(\Omega)} \\
& \quad \leq\left\|\boldsymbol{\omega} \times\left(\mathbf{v}-\mathbf{v}^{\prime}\right)\right\|_{L_{2}(\Omega)}+\left\|\left(\boldsymbol{\omega}-\boldsymbol{\omega}^{\prime}\right) \times \mathbf{v}^{\prime}\right\|_{L_{2}(\Omega)} \\
& \quad \leq\left\|\mathbf{v}-\mathbf{v}^{\prime}\right\|_{L_{4}(\Omega)}\left\|\nabla \mathbf{v}^{\prime}\right\|_{L_{4}(\Omega)}+\|\mathbf{v}\|_{L_{4}(\Omega)}\left\|\nabla\left(\mathbf{v}-\mathbf{v}^{\prime}\right)\right\|_{L_{4}(\Omega)} \\
& \quad \leq c\left\|A^{\frac{4}{9}} \mathbf{v}\right\|_{L_{2}(\Omega)}\left\|A^{\frac{8}{9}}\left(\mathbf{v}-\mathbf{v}^{\prime}\right)\right\|_{L_{2}(\Omega)}+c \| A^{\frac{4}{9}\left(\mathbf{v}-\mathbf{v}^{\prime}\right)\left\|_{L_{2}(\Omega)}\right\| A^{\frac{8}{9}} \mathbf{v}^{\prime} \|_{L_{2}(\Omega)}} .
\end{aligned}
$$

The estimate for $F_{2}(t)$ is even simpler

$$
\left\|F_{2}(t)-F_{2}\left(t^{\prime}\right)\right\|_{\mathcal{H}} \leq c\left\|\mathbf{f}(t)-\mathbf{f}\left(t^{\prime}\right)\right\|_{L_{2}(\Omega)} \leq c\left|t-t^{\prime}\right| .
$$

To verify $\left(\mathbf{C}_{3}\right)$ we check that for $\mathbf{u} \in D(A)$

$$
\begin{aligned}
\left(F_{1}(\mathbf{v}, t), \mathbf{u}\right) & =\int_{\Omega} \mathcal{P}(\boldsymbol{\omega} \times \mathbf{v}) \cdot \mathbf{u} \mathrm{d} x \leq\|\mathbf{u}\|_{L_{\infty}(\Omega)}\|\mathbf{v}\|_{L_{2}(\Omega)}\|\nabla \mathbf{v}\|_{L_{2}(\Omega)} \\
& \leq c\|A \mathbf{u}\|_{L_{2}(\Omega)}\|\mathbf{v}\|_{L_{2}(\Omega)}\left\|A^{\frac{1}{2}} \mathbf{v}\right\|_{L_{2}(\Omega)} .
\end{aligned}
$$

Let $\mathbf{u}=A^{-1} \mathbf{w}$. Due to the symmetry of $A^{-1}$ we have

$$
\left(A^{-1} F_{1}(\mathbf{v}, t), \mathbf{w}\right) \leq c\|\mathbf{w}\|_{L_{2}(\Omega)}\|\mathbf{v}\|_{L_{2}(\Omega)}\left\|A^{\frac{1}{2}} \mathbf{v}\right\|_{L_{2}(\Omega)},
$$

thus

$$
\left\|A^{-1} F_{1}(\mathbf{v}, t)\right\|_{\mathcal{H}} \leq c\|\mathbf{v}\|_{L_{2}(\Omega)}\left\|A^{\frac{1}{2}} \mathbf{v}\right\|_{L_{2}(\Omega)}
$$

Likewise

$$
\left(F_{2}(t), \mathbf{u}\right)=\int_{\Omega} \mathcal{P} \mathbf{f} \cdot \mathbf{u} \mathrm{d} x \leq c\|\mathbf{u}\|_{L_{\infty}(\Omega)}\|\mathbf{f}\|_{L_{1}(\Omega)} \leq c\|A \mathbf{u}\|_{L_{2}(\Omega)}\|\mathbf{f}\|_{L_{1}(\Omega)}
$$

and

$$
\left(A^{-1} F_{2}(t), \mathbf{w}\right) \leq c\|\mathbf{w}\|_{L_{2}(\Omega)}\|\mathbf{f}\|_{L_{1}(\Omega)},
$$

therefore

$$
\left\|A^{-1} F_{2}(t)\right\|_{\mathcal{H}} \leq c\|\mathbf{f}\|_{L_{1}(\Omega)} .
$$

We have completed the verification of all conditions. Using Lemma 5.4 we get the existence of weak solution, which are regular for almost all times and the existence of short-time strong solutions for any data or long-time strong solutions for small data.

For the general case, i.e. $\Pi \neq 0$, we follow the idea from Sect. 4. First, we introduce an auxiliary function $\tilde{\Pi}$ such that

$$
\begin{array}{ll}
-\Delta \tilde{\Pi}=0 & \text { in } \Omega, \\
\frac{\partial \tilde{\Pi}}{\partial \mathbf{n}}=0 & \text { on } \Gamma_{\text {lat }}, \\
\Pi=\Pi_{\text {in,out }} & \text { on } \Gamma_{\text {in,out }} .
\end{array}
$$

One can easily prove the existence of weak solutions to the above problem. They satisfy

$$
\|\tilde{\Pi}\|_{L i p\left(0, T ; H^{1}(\Omega)\right)} \leq c\left\|\Pi_{\text {in,out }}\right\|_{L i p\left(0, T ; H^{\frac{1}{2}}\left(\Gamma_{\text {in,out }}\right)\right)} .
$$


Next, we consider the pair $(\mathbf{v}, \psi)$, where $\psi=\Pi-\tilde{\Pi}$. This pair solves

$$
\begin{array}{ll}
\mathbf{v}_{, t}+\boldsymbol{\omega} \times \mathbf{v}+\nabla \psi-\nu \Delta \mathbf{v}=\mathbf{f}-\nabla \tilde{\Pi}=: \mathbf{G} & \text { in } \Omega^{T}=\Omega \times(0, T) \\
\operatorname{div} \mathbf{v}=0 & \text { in } \Omega^{T}, \\
\psi=0 & \text { on } \Gamma_{\text {in }, \text { out }}, \\
\mathbf{v}_{\text {tan }}=\mathbf{0} & \text { on } \Gamma, \\
\mathbf{v} \cdot \mathbf{n}=0 & \text { on } \Gamma_{\text {lat }}, \\
\left.\mathbf{v}\right|_{t=0}=\mathbf{v}_{0} & \text { on } \Omega \times\{t=0\} .
\end{array}
$$

If $\mathbf{f} \in \operatorname{Lip}\left(0, T ; L_{2}(\Omega)\right)$, then (26) ensures that $\mathbf{G} \in \operatorname{Lip}\left(0, T ; L_{2}(\Omega)\right)$. Utilizing Lemma 5.5 to (27) we conclude Theorem 2 .

Acknowledgements. The authors wish to express their thanks to the reviewer for their comments that helped improve the paper. The first author is grateful to the University of Iowa for the hospitality during his visit, when main parts of this paper were prepared.

\section{Compliance with Ethical Standards}

Conflict of interest The authors declare that they have no conflict of interest.

Open Access. This article is licensed under a Creative Commons Attribution 4.0 International License, which permits use, sharing, adaptation, distribution and reproduction in any medium or format, as long as you give appropriate credit to the original author(s) and the source, provide a link to the Creative Commons licence, and indicate if changes were made. The images or other third party material in this article are included in the article's Creative Commons licence, unless indicated otherwise in a credit line to the material. If material is not included in the article's Creative Commons licence and your intended use is not permitted by statutory regulation or exceeds the permitted use, you will need to obtain permission directly from the copyright holder. To view a copy of this licence, visit http://creativecommons.org/licenses/by/4.0/.

Publisher's Note Springer Nature remains neutral with regard to jurisdictional claims in published maps and institutional affiliations.

\section{References}

[1] Conca, C., Murat, F., Pironneau, O.: The Stokes and Navier-Stokes equations with boundary conditions involving the pressure. Jpn. J. Math. (N.S.) 20(2), 279-318 (1994)

[2] Pironneau, O.: Conditions aux limites sur la pression pour les équations de Stokes et de Navier-Stokes. C. R. Acad. Sci. Paris Sér. I Math. 303(9), 403-406 (1986)

[3] Heywood, J.G., Rannacher, R., Turek, S.: Artificial boundaries and flux and pressure conditions for the incompressible Navier-Stokes equations. Int. J. Numer. Methods Fluids 22(5), 325-352 (1996). https://doi.org/10.1002/ (SICI)1097-0363(19960315)22:5〈325::AID-FLD307〉3.0.CO;2-Y

[4] Becker, R., Capatina, D., Luce, R., Trujillo, D.: Finite element formulation of general boundary conditions for incompressible flows. Comput. Methods Appl. Mech. Eng. 295, 240-267 (2015). https://doi.org/10.1016/j.cma.2015.07.002

[5] Gresho, P.M.: Some current CFD issues relevant to the incompressible Navier-Stokes equations. Comput. Methods Appl. Mech. Eng. 87(2-3), 201-252 (1991). https://doi.org/10.1016/0045-7825(91)90006-R

[6] Korobkov, M.V., Piletskas, K., Pukhnachëv, V.V., Russo, R.: The flux problem for Navier-Stokes equations, Uspekhi Mat. Nauk 69(6(420)), 115-176 (2014). translation in: Russian Math. Surveys 69(6), 1065-1122 (2014). https://doi.org/ $10.4213 / \mathrm{rm} 9616$

[7] Specovius-Neugebauer, M.: Approximation of the Stokes Dirichlet problem in domains with cylindrical outlets. SIAM J. Math. Anal. 30(3), 645-677 (1999). https://doi.org/10.1137/S0036141097325083

[8] Nazarov, S.A., Specovius-Neugebauer, M., Videman, J.H.: Nonlinear artificial boundary conditions for the Navier-Stokes equations in an aperture domain. Math. Nachr. 265, 24-67 (2004). https://doi.org/10.1002/mana.200310135

[9] Rencławowicz, J., Zajączkowski, W.: Existence of global weak solutions for Navier-Stokes equations with large flux. J. Differ. Equ. 251(3), 688-707 (2011). https://doi.org/10.1016/j.jde.2011.04.024

[10] Rencławowicz, J., Zajączkowski, W.: Global nonstationary Navier-Stokes motion with large flux. SIAM J. Math. Anal. 46(4), 2581-2613 (2014). https://doi.org/10.1137/120880823

[11] Bruneau, C.-H., Fabrie, P.: New efficient boundary conditions for incompressible Navier-Stokes equations: a well-posedness result. RAIRO Modél. Math. Anal. Numér. 30(7), 815-840 (1996). https://doi.org/10.1051/m2an/ 1996300708151

[12] Feistauer, M., Neustupa, T.: On the existence of a weak solution of viscous incompressible flow past a cascade of profiles with an arbitrarily large inflow. J. Math. Fluid Mech. 15(4), 701-715 (2013). https://doi.org/10.1007/s00021-013-0135-4 
[13] Kučera, P., Skalák, Z.: Local solutions to the Navier-Stokes equations with mixed boundary conditions. Acta Appl. Math. 54(3), 275-288 (1998). https://doi.org/10.1023/A:1006185601807

[14] Kračmar, S., Neustupa, J.: A weak solvability of a steady variational inequality of the Navier-Stokes type with mixed boundary conditions, In: Proceedings of the Third World Congress of Nonlinear Analysts, Part 6 (Catania, 2000 ), vol. 47, pp. 4169-4180 (2001). https://doi.org/10.1016/S0362-546X(01)00534-X

[15] Bothe, D., Kashiwabara, T., Köhne, M.: Strong well-posedness for a class of dynamic outflow boundary conditions for incompressible Newtonian flows. J. Evol. Equ. 17(1), 131-171 (2017). https://doi.org/10.1007/s00028-016-0352-4

[16] Bègue, C., Conca, C., Murat, F., Pironneau, O.: Les équations de Stokes et de Navier-Stokes avec des conditions aux limites sur la pression, In: Nonlinear partial differential equations and their applications. Collège de France Seminar, Vol. IX (Paris, 1985-1986), vol. 181 of Pitman Res. Notes Math. Ser., Longman Sci. Tech., Harlow, pp. 179-264 (1988)

[17] Bègue, C., Conca, C., Murat, F., Pironneau, O.: À nouveau sur les équations de Stokes et de Navier-Stokes avec des conditions aux limites sur la pression. C. R. Acad. Sci. Paris Sér. I Math. 304(1), 23-28 (1987)

[18] Conca, C., Parés, C., Pironneau, O., Thiriet, M.: Navier-Stokes equations with imposed pressure and velocity fluxes. Int. J. Numer. Methods Fluids 20(4), 267-287 (1995). https://doi.org/10.1002/fld.1650200402

[19] Bernard, J.M.: Non-standard Stokes and Navier-Stokes problems: existence and regularity in stationary case. Math. Methods Appl. Sci. 25(8), 627-661 (2002). https://doi.org/10.1002/mma.260

[20] Bernard, J.M.: Time-dependent Stokes and Navier-Stokes problems with boundary conditions involving pressure, existence and regularity. Nonlinear Anal. Real World Appl. 4(5), 805-839 (2003). https://doi.org/10.1016/ S1468-1218(03)00016-6

[21] Kim, T., Cao, D.: Some properties on the surfaces of vector fields and its application to the Stokes and Navier-Stokes problems with mixed boundary conditions. Nonlinear Anal. 113, 94-114 (2015). https://doi.org/10.1016/j.na.2014.09. 017

[22] Kučera, P.: Basic properties of solution of the non-steady Navier-Stokes equations with mixed boundary conditions in a bounded domain. Ann. Univ. Ferrara Sez. VII Sci. Mat. 55(2), 289-308 (2009). https://doi.org/10.1007/ s11565-009-0082-4

[23] Kračmar, S., Neustupa, J.: Modeling of the unsteady flow through a channel with an artificial outflow condition by the Navier-Stokes variational inequality. Math. Nachr. 291(11-12), 1801-1814 (2018). https://doi.org/10.1002/mana. 201700228

[24] Kim, T.: Regularity of solutions to the Navier-Stokes equations with a nonstandard boundary condition. Acta Math. Appl. Sin. Engl. Ser. 31(3), 707-718 (2015). https://doi.org/10.1007/s10255-015-0497-x

[25] Grisvard, P.: Elliptic Problems in Nonsmooth Domains. Monographs and Studies in Mathematics, vol. 24. Pitman (Advanced Publishing Program), Boston (1985)

[26] Agmon, S., Douglis, A., Nirenberg, L.: Estimates near the boundary for solutions of elliptic partial differential equations satisfying general boundary conditions. I. Commun. Pure Appl. Math. 12, 623-727 (1959)

[27] Ziemer, W.P.: Weakly Differentiable Functions, Vol. 120 of Graduate Texts in Mathematics. Springer, New York (1989), Sobolev Spaces and Functions of Bounded Variation. https://doi.org/10.1007/978-1-4612-1015-3

[28] Galdi, G.P.: An introduction to the mathematical theory of the Navier-Stokes equations. Springer Monographs in Mathematics, 2nd Edn. Springer, New York (2011), Steady-State Problems. https://doi.org/10.1007/978-0-387-09620-9

[29] Kučera, P., Skalák, Z.: Local solutions to the Navier-Stokes equations with mixed boundary conditions. Acta Appl. Math. 54(3), 275-288 (1998). https://doi.org/10.1023/A:1006185601807

[30] Beneš, M., Kučera, P.: Solutions of the Navier-Stokes equations with various types of boundary conditions. Arch. Math. (Basel) 98(5), 487-497 (2012). https://doi.org/10.1007/s00013-012-0387-x

[31] Brezis, H.: Functional Analysis, Sobolev Spaces and Partial Differential Equations, Universitext. Springer, New York (2011)

[32] Zeidler, E.: Applied Functional Analysis, Vol. 108 of Applied Mathematical Sciences. Springer, New York (1995), Applications to Mathematical Physics

[33] Cholewa, J.W., Dłotko, T.: Global Attractors in Abstract Parabolic Problems. Vol. 278 of London Mathematical Society Lecture Note Series. Cambridge University Press, Cambridge (2000). https://doi.org/10.1017/CBO9780511526404

[34] Ströhmer, G.: A remark about a Galerkin method, In: Singularities and Differential Equations (Warsaw, 1993), Vol. 33 of Banach Center Publ., Polish Acad. Sci., Warsaw, pp. 365-367 (1996). http://matwbn.icm.edu.pl/ksiazki/bcp/bcp33/ bcp33132.pdf

[35] Ströhmer, G.: An existence result for partially regular weak solutions of certain abstract evolution equations, with an application to magnetohydrodynamics. Math. Z. 213(3), 373-385 (1993). https://doi.org/10.1007/BF03025726

Bernard Nowakowski

Institute of Mathematics and Cryptology

Military University of Warsaw

Warsaw

Poland

e-mail: bernard.nowakowski@wat.edu.pl
Gerhard Ströhmer

College of Liberal Arts and Sciences, Mathematics

Department

The University of Iowa

Iowa City IA

USA

e-mail: gerhard-strohmer@uiowa.edu

(accepted: June 15, 2020; published online: September 4, 2020) 